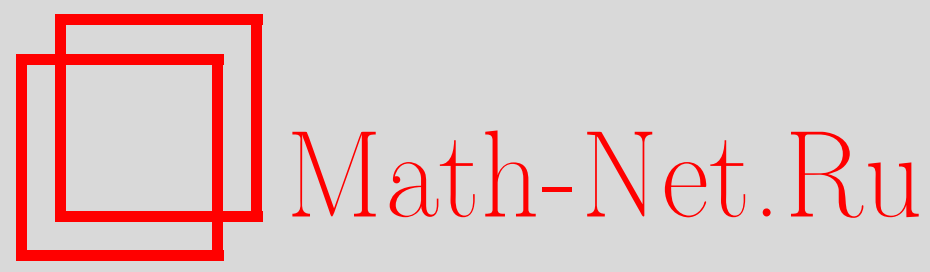

С. Л. Зиглин, О первых интегралах групп комплексных линейных преобразований и натуральных механических систем с однородным потенциалом, Матем. заметки, 2001, том 70, выпуск 6, 839-844

DOI: https://doi.org/10.4213/mzm796

Использование Общероссийского математического портала Math-Net.Ru подразумевает, что вы прочитали и согласны с пользовательским соглашением http://www.mathnet.ru/rus/agreement

Параметры загрузки:

IP: 54.80 .97 .219

26 апреля 2023 г., 13:37:08

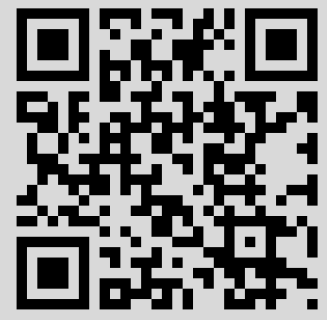




\section{О ПЕРВЫХ ИНТЕГРАЛАХ ГРУПП КОМПЛЕКСНЫХ ЛИНЕЙНЫХ ПРЕОБРАЗОВАНИЙ И НАТУРАЛЬНЫХ МЕХАНИЧЕСКИХ СИСТЕМ С ОДНОРОДНЫМ ПОТЕНЦИАЛОМ}

\section{С. Л. Зиглин}

Исследуется вопрос о существовании рациональных первых интегралов групп комплексных линейных преобразований. Полученные результаты применяются к натуральньм механическим системам с однородным потенциалом, в частности, к задаче Суслова о движении твердого тела около неподвижной точки с неголономной связью в случае Козлова при нулевой постоянной энергии.

Библиографоия: 14 названий.

1. Общая теория. Пусть $L$ - конечномерное комплексное линейное пространство, $G$-подгруппа группы его линейных преобразований, $H$-подгруппа группы $G$ конечного индекса.

Лемма. Числа функиионально независимых рачиональных первых интегралов zpynn $G$ u $H$ coвnaдают.

ДокАЗАТЕЛЬСтво. Пусть $f_{1}, \ldots, f_{r}$ - функционально независимые рациональные первые интегралы группы $H$. Тогда для любых $g, g^{\prime} \in G$ таких, что $g \in H g^{\prime}$, имеем $f_{1} \circ g=f_{1} \circ g^{\prime}$; следовательно, число функций $f_{1} \circ g$, где $g \in G$, конечно. Обозначим их через $F_{1}, \ldots, F_{k}$.

Рассмотрим первые интегралы $\varphi_{i}=\sum_{j=1}^{k} F_{j}^{i}(i=1, \ldots, k)$ группы $G$. Имеем

$$
\left(\begin{array}{c}
d \varphi_{1} \\
\vdots \\
d \varphi_{k}
\end{array}\right)=\frac{\partial\left(\varphi_{1}, \ldots, \varphi_{k}\right)}{\partial\left(F_{1}, \ldots, F_{k}\right)}\left(\begin{array}{c}
d F_{1} \\
\vdots \\
d F_{k}
\end{array}\right), \quad \operatorname{det} \frac{\partial\left(\varphi_{1}, \ldots, \varphi_{k}\right)}{\partial\left(F_{1}, \ldots, F_{k}\right)}=k ! \prod_{i<j \leqslant k}\left(F_{i}-F_{j}\right) .
$$

Пусть в точке $x \in L$ значения функций $F_{1}, \ldots, F_{k}$ попарно различны и дифференциалы $d f_{1}, \ldots, d f_{r}$ линейно независимы. Тогда дифференциал $d_{x} f_{1}$ является линейной комбинацией дифференциалов $d_{x} \varphi_{1}, \ldots, d_{x} \varphi_{k}$ и существует $i(1 \leqslant i \leqslant k)$ такое, что дифференциалы $d_{x} \varphi_{i}, d_{x} f_{2}, \ldots, d_{x} f_{r}$ линейно независимы.

Заменим первый интеграл $f_{1}$ групшы $H$ на первый интеграл $\varphi_{i}$ группы $G$, затем аналогично поступим с $f_{2}$ и т.д. В результате получим систему $r$ функционально независимых рациональных первых интегралов группы $G$, что и требовалось доказать.

Пусть $G$-подгруппа групшы линейных преобразований двумерной комплексной плоскости $L^{2}$ с определителями, являющимися корнями фиксированной степени из единицы.

Из теоремы 3.3 работы [1] вытекает следующая теорема, совпадающая при $G \subset$ $\mathrm{SL}(2, \mathbb{C})$ со следствием 3.4 из [1]. 
Теорема 1. Для существования у группы $G$ рационального первого интеграла необходимо и достаточно выполнение одного из следующих условий:

1) все преобразования группы $G$ имеют общий собственный вектор, а их собственные значения являются корнями из единицы фиксированной степени;

2) существует пара прямых, проходящих через $0 \in L^{2}$, обгединение которых инвариантно относительно $G$;

3) группа $G$ конечна.

Дадим независимое

ДОКАЗАТЕЛЬСТво ТЕОРЕМЫ 1 . Достаточность условий 1), 2) очевидна. Достаточность условия 3) следует из леммы.

Докажем необходимость. Пусть $f$ - рациональный первьй интеграл групшы $G$. Не ограничивая общности, будем считать его однородным. Представим его в виде несократимой дроби. Если множество прямых, на которых обращается в нуль ее числитель или знаменатель, состоит из одного элемента, то в соответствующих координатах имеем $f=x^{n}, n \in \mathbb{Z} \backslash\{0\}$, и вьполняется условие 1$)$; если из двух - то условие 2); если более - то условие 3 ) (так как одномерное проективное преобразование определяется образами трех точек (см., например, [2]), то проективизация групшы $G$, а значит, и она сама, конечна), что и требовалось доказать.

Теорема 2. Для существования у группы $G$ рационального первого интеграла необходимо и достаточно выполнение одного из условий: 1), 2) или

$\left.3^{\prime}\right)$ проективизачия группы $G$ изоморфна әруппе вращений куба, тетраэдра или икосаэдра.

СлЕДСТВИЕ. Если хотя бы один әлемент проективизации группь $G$ имеет порядок больие пяти, то для существования рационального первого интеграла әруппы $G$ необходимо и достаточно выполнение условий 1) или 2).

ДокАЗАТЕЛЬСТво тЕорЕмы 2. По теореме Клейна (см., например, [3], [4]) действие конечной подгруппы проективной групш комплексной проективной прямой сопряжено действию либо конечной циклической подгруппы группы вращения сферы, либо подгрупшы этой групш, порожденной вращениями диэдра или многогранников, указанных в условии $3^{\prime}$ ) с центром в центре сферы. Но в первых двух случаях оно имеет инвариантное множество, состоящее из двух точек, следовательно, вьполняется условие 2). Теорема доказана.

2. Натуральные механические системы с однородным потенциалом. Пусть $D$ - область в $n$-мерном комплексном координатном пространстве $\mathbb{C}^{n}$, инвариантная относительно умножения на ненулевые комплексные числа и такая, что $\operatorname{Re} D=D \cap \mathbb{R}^{n} \neq \varnothing$; $V$ - вещественно-аналитическая однородная функция в $D$ целой степени $k$.

Будем использовать следующие обозначения. Если $x=\left(x_{1}, \ldots, x_{n}\right) \in \mathbb{C}^{n}, y=\left(y_{1}\right.$, $\left.\ldots, y_{n}\right) \in \mathbb{C}^{n}$, то $\langle x, y\rangle=\sum x_{i} y_{i}, x^{2}=\langle x, x\rangle$.

Рассмотрим, следуя [5], [6], гамильтонову систему

$$
\begin{gathered}
\dot{q}=\frac{\partial H}{\partial p}, \quad \dot{p}=-\frac{\partial H}{\partial q}, \quad q \in D, \quad p \in \mathbb{C}^{n}, \\
H=\frac{1}{2} p^{2}+V(q) .
\end{gathered}
$$


Пусть $c \in \operatorname{Re} D$ - ненулевое решение системы уравнений

$$
c=\frac{\partial V}{\partial q}(c) .
$$

ЗАмЕчАниЕ. При $k=0$ таких решений нет, так как при вешественных $q$ имеем $q \perp \partial V / \partial q$.

Рассмотрим прямолинейное решение $q=c \phi(t), p=c \dot{\phi}(t)$ системы $(1)$, где $\phi$ - решение дифференциального уравнения

$$
\ddot{\phi}+\phi^{k-1}=0
$$

с начальньм условием $\phi(0)=1, \dot{\phi}(0)=0$.

Как показано в [7] (cp. [5], [6]), если система (1) имеет в какой-либо области, содержашей комплексную фазовую кривую Г этого решения, $n$ функционально независимых мероморфных первых интегралов в инволюции, то групша монодромии каждого из уравнений

$$
\dot{\xi}+\lambda_{i} \phi^{k-2}(t) \xi=0, \quad i=1, \ldots, n,
$$

где $\lambda_{i}$ - собственные значения матрицы $\partial^{2} V / \partial q^{2}(c)$, т.е. образ естественного антипредставления фундаментальной групш фазовой кривой Г в какой-либо точке в групу линейных преобразований комплексной плоскости $\mathbb{C}^{2}$ под действием уравнения $(3)$, имеет рациональньй первьй интеграл.

При $k= \pm 2$ группа монодромии уравнения

$$
\ddot{\xi}+\lambda \phi^{k-2}(t) \xi=0
$$

имеет линейньй или квадратичньй первьй интеграл [7] (при $k=2$ это очевидно; см. также ниже).

ТЕОрема 3. Для существования у группы монодромии уравнения (4) рационального первого интеграла необходимо и достаточно выполнение одного из следующих условий: при $k= \pm 3$ u $m \in \mathbb{Z}$

$$
\begin{aligned}
\lambda & =(m k-1)(2 m-1), \\
\lambda & =m(2 m k-k+2), \\
\lambda & =\frac{1}{8 k}\left[(2 m+1)^{2} k^{2}-(k-2)^{2}\right], \\
\lambda & =\frac{1}{8 k}\left[4 k^{2}\left(m+\frac{1}{3}\right)^{2}-(k-2)^{2}\right], \\
\lambda & =\frac{1}{8 k}\left[4 k^{2}\left(m+\frac{2}{5}\right)^{2}-(k-2)^{2}\right], \\
\lambda & =\frac{1}{8 k}\left[4 k^{2}\left(m+\frac{1}{4}\right)^{2}-(k-2)^{2}\right], \\
\lambda & =\frac{1}{8 k}\left[4 k^{2}\left(m+\frac{1}{5}\right)^{2}-(k-2)^{2}\right] ;
\end{aligned}
$$

при $k= \pm 4$ - одного из условий (5)-(8); при $k= \pm 5$ - одного из условий (5)-(9); при $|k|>5$ - одного из условий (5)-(7); при $k= \pm 1-$ условия (5). 
СлЕДСТВИЕ [8, теорема 5.1]. Если система (1) имеет в какой-либо области, содержащей фазовую кривую $\Gamma, n$ функиионально независимых мероморфных первых интегралов в инволюиии, то каждое из чисел $\lambda=\lambda_{i}$ удовлетворяет одному из условий (5)-(11).

ЗАмЕчАниЕ. В [5], [6] указаны необходимые условия существования полиномиального первого интеграла групшы монодромии уравнения (4) при $k \neq 0, \pm 2$. При $k= \pm 1$ условия [5] совпадают с (5).

ДоКАЗАТЕЛЬСТво ТЕОРЕМЫ 3 . При $k \neq 0$ уравнение (4) эквивалентно гипергеометрическому уравнению [5]

$$
z(1-z) \frac{d^{2} \xi}{d z^{2}}+(c-(a+b+1) z) \frac{d \xi}{d z}-a b \xi=0
$$

где

$$
z=(\phi(t))^{k}, \quad a+b=\frac{1}{2}-\frac{1}{k}, \quad a b=-\frac{\lambda}{2 k}, \quad c=1-\frac{1}{k} .
$$

Группа монодромии уравнения (4) совпадает с подгруппой группы монодромии уравнения (12), порожденной петлями, обходящими точку $z=0$ число раз, кратное $k$, а точку $z=1$ - четное число раз [5]. Эта подгруппа имеет конечньй индекс, следовательно, числа функционально независимых рациональных первых интегралов групा монодромии уравнений (4) и (12) совпадают.

При $a+b-c \notin \mathbb{Z}$ групша монодромии $G$ уравнения (12) в соответствующем базисе имеет образуюшие [9, п. 15.93]

$$
g_{1}=\left(\begin{array}{cc}
\frac{A B}{C} & 0 \\
0 & 1
\end{array}\right), \quad g_{0}=\left(\begin{array}{cc}
C-\frac{(A-1)(B-1)}{A B / C-1} & \beta \\
\gamma & 1+\frac{(A-1)(B-1)}{A B / C-1}
\end{array}\right) \text {, }
$$

где

$$
\begin{gathered}
\beta \gamma=-\frac{(A-1)(B-1)(A-C)(B-C)}{C(A B / C-1)^{2}}, \\
A=\exp (-2 \pi i a), \quad B=\exp (-2 \pi i b), \quad C=\exp (-2 \pi i c) .
\end{gathered}
$$

Из теоремы 1 п. 1 следует, что при $k \neq \pm 2$ эта группа имеет рациональньй первьй интеграл тогда и только тогда, когда вьполняется одно из следующих условий:

1) преобразование $g_{0}$ сохраняет одну из собственных прямых преобразования $g_{1}$;

2) преобразование $g_{1}$ переставляет пару собственных прямых преобразования $g_{0}$;

3) группа $G$ конечна.

Условие 1) эквивалентно условию $\beta \gamma=0$, т.е. совокупности условий $A=C, B=C$, $A=1, B=1$ или, что то же, условий (5), (6). При $k= \pm 1$ эти условия между собой совпадают.

Условие 2 ) при $k= \pm 1$ выполняется только при $\beta=\gamma=0$. При $k \neq \pm 1$ оно эквивалентно условию Trace $g_{0} g_{1}=A+B=0$ или, что то же, условию (7). 
При $\beta \gamma \neq 0$, как следует из таблицы Шварца [9]-[11], случаев интегрируемости гипергеометрического уравнения в алгебраических функциях или, что то же, случаев конечности его групш монодромии, условие 3) вьполняется только в следующих случаях:

$$
\pm(a-b)=\frac{1}{2}+m, \quad m \in \mathbb{Z}
$$

что эквивалентно условию (7);

$$
|k|=3,4,5 \quad \text { и } \quad \pm(a-b)=\frac{1}{3}+m, \quad m \in \mathbb{Z},
$$

что эквивалентно условию (8);

$$
|k|=3,5 \quad \text { и } \quad \pm(a-b)=\frac{2}{5}+m, \quad m \in \mathbb{Z},
$$

что эквивалентно условию (9);

$$
|k|=3 \quad \text { и } \quad \pm(a-b)=\frac{1}{4}+m, \quad m \in \mathbb{Z},
$$

что эквивалентно условию (10); или

$$
\pm(a-b)=\frac{1}{5}+m, \quad m \in \mathbb{Z},
$$

что эквивалентно условию (11). Теорема доказана.

ЗАмЕчАнИЕ. Из приведенного доказательства следует независимое доказательство существования квадратичного первого интеграла группы монодромии уравнения (4) при $k= \pm 2$. Действительно, при этих значениях $k$ преобразования $g_{0}, g_{1}$ сохраняют объединение собственных прямых преобразования $g_{0} g_{1}$. Если таких прямых больше двух, то они сохраняют объединение двух, откуда следует высказанное утверждение.

3. Задача Суслова. Рассмотрим задачу Суслова [12] о движении твердого тела около неподвижной точки с неголономной связью - проекция кинетического момента на направление, неподвижное в системе координат, связанной с телом, равна нулю - в случае [13], когда это направление совпадает с направлением одной из главных осей инерции в точке подвеса, тело находится в однородном поле тяжести и центр тяжести лежит на указанной оси.

Как показано в [13], в этом случае ограничение системы на поверхность уровня $h$ интеграла энергии эквивалентно ограничению системы с гамильтонианом

$$
F=\frac{1}{2}\left(p_{1}^{2}+p_{2}^{2}\right)+V, \quad V=\frac{1}{2}\left(h-\frac{q_{1}^{2}}{2 I_{1}}-\frac{q_{2}^{2}}{2 I_{2}}\right)^{2},
$$

(здесь $I_{1}, I_{2}$ - моменты инерции тела относительно двух других главных осей инерции в точке подвеса) на поверхность $S$ уровня гамильтониана $F=\mu^{2} / 2$, где $\mu$-параметр Пуанкаре - произведение веса тела на расстояние от центра тяжести до точки подвеса.

Как показано в [14], если хотя бы одно из чисел $\sqrt{1+8 \alpha}, \sqrt{1+8 / \alpha}$, где $\alpha=I_{1} / I_{2}$, иррационально, то при $h=0$ ограничение системы с гамильтонианом $F$ на поверхность $S$ не имеет (непостоянного) мероморфного первого интеграла. 
Теорема 4. Ограничение системы с гамильтонианом $F$ на поверхность $S$ имеет мероморфный первый интеграл только в случае $\alpha=1$ (аналоге случая Лагранжа движения тяжелого твердого тела около неподвижной точки без дополнительных связей).

ДоказАтельство. Введем обозначения: $p=\left(p_{1}, p_{2}\right), q=\left(q_{1}, q_{2}\right), D=\left\{(p, q) \in \mathbb{C}^{4} \mid\right.$ $\left.F(p, q) \notin \overline{\mathbb{R}}^{-}\right\}$, где $\overline{\mathbb{R}}^{-}$- замькание отрицательной части вещественной оси. Выберем единищы измерения так, что $\mu=\sqrt{2}$. Рассмотрим ретракцию $r: D \rightarrow S, r:(p, q) \mapsto$ $\left(p / \sqrt{H}, q / H^{1 / 4}\right)$. Предположим, что ограничение системы с гамильтонианом $F$ на поверхность $S$ имеет непостоянньй мероморфный первый интеграл $f$. Тогда в области $D$ она имеет мероморфньй первый интеграл $f$ о $r$, функционально независимьй от $F$.

Уравнение $\partial V / \partial q(c)=c$ имеет решение $c=\left(c_{1}, c_{2}\right), c_{1}=\sqrt{2} I_{1}, c_{2}=0$. Собственные значения матрицы $\partial^{2} V / \partial q^{2}(c)$ равны 3 и $1 / I_{2}=\alpha$. Из следствия, приведенного в п. 2 , получаем, что $\alpha=m / 72$, где $m$ целое. Аналогично $1 / \alpha=n / 72$, где $n$ целое, следовательно, $72^{2}: m$, откуда $\alpha= \pm 2^{k} 3^{l}$, где $k, l$ целые, $|k| \leqslant 3,|l| \leqslant 2$. При всех таких значениях $\alpha$, кроме $\alpha=1$, хотя бы одно из чисел $\sqrt{1+8 \alpha}, \sqrt{1+8 / \alpha}$ иррационально. Теорема доказана.

Автор глубоко благодарен Д. В. Аносову, Ю. С. Ильяшенко, В. В. Козлову, А. И. Нейштадту и А.И. Овсеевичу за полезные обсуждения и Ж. Ж. Моралесу-Руизу за присланную книгу [8] и любезные разъяснения.

\section{СПИСОК ЦИТИРОВАННОЙ ЛИТЕРАТУРЫ}

[1] Churchill R. C., Rod D. L. On the determination of Ziglin monodromy groups // SIAM J. Math. Anal. 1991. V. 22. №6. P. 1790-1802.

[2] Кострикин А. И., Манин Ю. И. Линейная алгебра и геометрия. М.: Наука, 1986.

[3] Клейн $\Phi$. Лекции о развитии математики в ХІХ столетии. Т. 1. М.-Л.: ОНТИ, 1937.

[4] Форд Р. Автоморфные функции. М.-Л.: ОНТИ, 1936.

[5] Yoshida H. A criterion for non-existence of an additional integral in Hamiltonian systems with homogeneous potential // Phys. D. 1987. V. 29. №1, 2. P. 128-142.

[6] Yoshida H. A criterion for non-existence of an additional analytic integral in Hamiltonian systems with $n$ degrees of freedom // Phys. Lett. A. 1989. V. 141. №3, 4. P. 108-112.

[7] Зиглин С. Л. Об интегралах в инволюции групп линейных симплектических преобразований и натуральных механических систем с однородным потенциалом // Функцион. анализ и его прилож. 2000. Т. 34. № 3. С. 26-36.

[8] Morales-Ruis J. J. Differential Galois Theory and Non-Integrability of Hamiltonian Systems. Progress in Math. V. 179: Birkhäuser, 1999.

[9] Айнс Э. Л. Обыкновенные дифференциальные уравнения. Харьков: ОНТИ, 1939.

[10] Бейтмен Г., Эрдейи Ф. Высшие трансцендентные функции. Т. 1. М.: Наука, 1973.

[11] Арнольд В.И., Ильяшенко Ю.С. Обькновенные дифференциальные уравнения // Итоги науки и техники. Современные проблемы математики. Фундаментальные направления. Т. 1. М.: ВИНИТИ, 1985. С. 7-149.

[12] Суслов Г. К. Теоретическая механика. М.-Л.: Гостехиздат, 1946.

[13] Козлов В.В. К теории интегрирования уравнений неголономной механики // Успехи механики. 1985. Т. 8. № 3. С. $85-107$.

[14] Зиглин С. Л. Об отсутствии дополнительного первого интеграла в одной задаче динамики твердого тела // Докл. АН СССР. 1987. Т. 292. № 4. С. 804-807. 